MATEC Web of Conferences 40, 01006 (2016)

DOI: $10.1051 /$ matecconf/20164001006

(c) Owned by the authors, published by EDP Sciences, 2016

\title{
Review of wide band-gap semiconductors technology
}

\author{
Haiwei Jin ${ }^{1}$, Li Qin ${ }^{1}$, Lan Zhang ${ }^{1}$, Xinlin Zeng ${ }^{1}$, Rui Yang ${ }^{1}$ \\ ${ }^{1}$ Library, China Defence Science and Technology Information Center, china
}

\begin{abstract}
Silicon carbide $(\mathrm{SiC})$ and gallium nitride $(\mathrm{GaN})$ are typical representative of the wide band-gap semiconductor material, which is also known as third-generation semiconductor materials. Compared with the conventional semiconductor silicon ( $\mathrm{Si}$ ) or gallium arsenide (GaAs), wide band-gap semiconductor has the wide band gap, high saturated drift velocity, high critical breakdown field and other advantages; it is a highly desirable semiconductor material applied under the case of high-power, high-temperature, high-frequency, anti-radiation environment. These advantages of wide band-gap devices make them a hot spot of semiconductor technology research in various countries. This article describes the research agenda of United States and European in this area, focusing on the recent developments of the wide band-gap technology in the US and Europe, summed up the facing challenge of the wide band-gap technology.
\end{abstract}

\section{INTRODUCTION}

Widely used of semiconductor technology in various military fields broke the traditional concept formed in thousa-

nds of years that weapons equipment's advantage is only the size, the number and the more of massive destruction, so that system of weapons become smaller, lighter, less power wasted, higher reliable, stronger operational effective and powerful. Military electronic equipment required to operate at high temperature, high radiation and other harsh environments, can detect small targets of long-distance and real-time process high-speed sensor data, and the operating frequency band beyond the ordinary commercial range. Thus, require for the military electronic equipment for semiconductor components is much higher than ordinary electronic equipment, its safety and reliability of components must be higher. It is worth noting that, using conventional semiconductor technology to produce electronic systems has been unable to meet the requirements of next generation military applications for volume, weight and higher reliability. Wide band-gap semiconductor device has advantage of high frequency, high power, high temperature and potential of resistance to harsh environments, so that provides a method to solve these problem.

Semiconductor of band gap greater than $2.2 \mathrm{eV}$ is defined as the wide band-gap semiconductor (WBGS), typical of wide band-gap semiconductor materials are silicon carbide ( $\mathrm{SiC})$ and gallium nitride $(\mathrm{GaN})$, these semiconductor materials are also known as thirdgeneration semiconductor material. In comparison with $\mathrm{Si}$ and $\mathrm{GaAs}$ as the representative of the second generation of semiconductor, wide band gap semiconductor has merits of wide band gap, high saturated drift velocity, high critical breakdown electric field. in order to highlight the advantages compared to $\mathrm{Si}$ and $\mathrm{GaAs}$ as the representative of the second generation of semiconductors. In recent years, as $\mathrm{SiC}$ single crystal growth technology and GaN Hetero-epitaxial technology continue to mature, wide band-gap semiconductor power device's development and application rise rapidly.

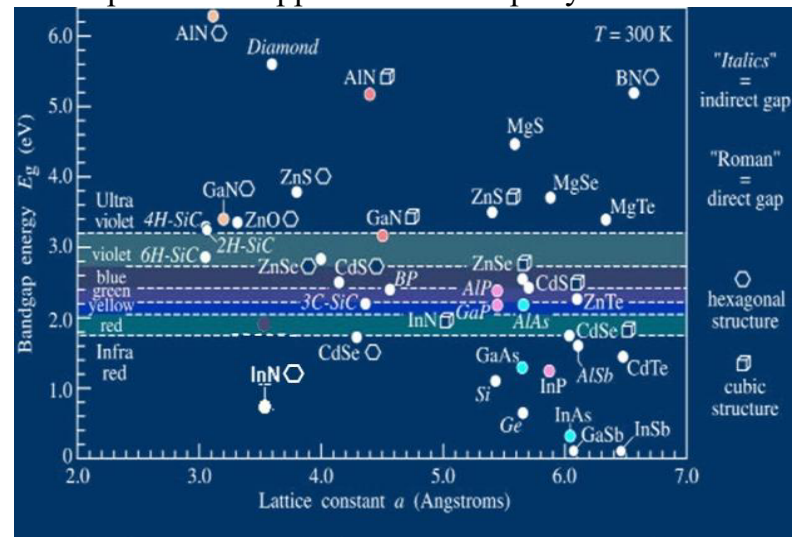

Figure 1. Wide band gap materials and their forbidden band width

In the start-up and push of wide band-gap semiconductor technology program (WBGSTI) of US Defense Advanced Research Projects Agency, many other research projects of Europe ESCAPEE and Japan NEDO, SiC, GaN and other wide band-gap semiconductor materials and devices research gained rapid development; a number of international semiconductor manufacturers have introduced highpower, high-frequency, high-temperature wide band-gap semiconductor products, whose applications are con- 
stantly expanded. In short, American and European countries will always put wide band-gap semiconductor technology on an extremely important strategic position, hesitate to invest heavily to implement a number of wide band gap semiconductor technology development plan in order to enhance the ability of military equipment, and have achieved good results. This article described the development of major initiatives in Europe and America in a wide band-gap semiconductor technology and the latest progress in the development and research in the device. It focused on the wide band-gap semiconductor technology development plan implemented in US, Europe and other developed countries and the far-reaching impact on military or other important facilities produce, summed up the facing challenge of the wide band-gap technology.

\section{AMERICAN WIDE BAND-GAP SEMICONDUCTOR TECHNOLOGY}

United States was the first country to start the wide bandgap semiconductor technology research, and many wellknown universities and research institutions, such as Raytheon, TriQuint, BAE, MIT, Cree, put a lot of manpower and material resources in the technology, and their research is most outstanding. DARPA hesitated to invest huge amounts of money to implement a number of wide band-gap semiconductor device and circuit technology development plans. DARPA aimed to enhance military radar, submarines and various other systems and equipment performance and reliability operated in harsh environment, and solved a number of technical bottlenecks of wide band-gap technology currently facing.

It is worth noting that, so far the largest investment, the largest participating institutions, the results of the most prominent and most influential program is counted the wide band-gap semiconductor technology initiative (WBGSTI), which launched by DARPA in 2002, lasted eight years, divided in three stages; the first phase from 2002 to 2004, focused on material development, achieve commercialization of 2-4 inches $\mathrm{SiC}$ substrate material, has been completed now; the second phase from 2005 to 2007, focused on device development, used wide band gap semiconductor material to product and demonstrate the RF power amplifier for improving its power-added rate, bandwidth and power density, ultimately achieved high-volume production of high reliability, highperformance microwave and millimeter-wave GaN-based devices; the third stage, from 2008 to 2009, developed GaN-based high reliable, high performance MMIC (monolithic microwave integrated circuit), and test their applications in a number of modules; this program cost hundreds millions of dollars.

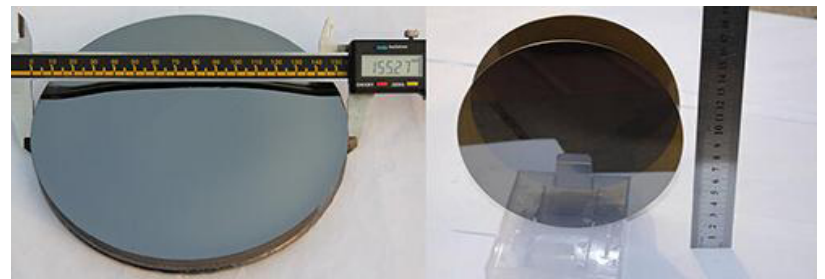

Figure 2. Six inchs silicon carbide crystal and single crystal substrate

With the support of DARPA, Raytheon achieved the world advanced level in the wide band-gap technical field, such as the first GaN MMIC produced by Raytheon; completed a historic first X- band GaN T / R (transmitter / receiver modules) verification, it included with a range of operating conditions, widely and successfully designed a confirmatory test to verify the GaN technology maturity, on this basis, completed a historic first X- band GaN TRIMM (final transmitter / receiver multichannel integration module) verification, confirming the GaN performance under the relevant operating conditions, including extensive tests in a relevant combination work environment, which including a operation test for a trial GaN array at 1000 hours in a laboratory, and conducted GaN array insert verification tests in a production radar, ready for TRIMM into the production stage, as a milestone in the success of the second phase of the WBGS project task; in 2013 due to successful completion of the Defense Production Act Title III GaN production improvement projects, it rewarded by office of the Secretary of Defense; through this project Raytheon's GaN production has increased at least 300 percent, the cost of monolithic microwave integrated circuits decreased at least $75 \%$. Currently GaN has become the Raytheon Company's core competitiveness, as well as technology pillars behind the major project, such as air, missile defense radar and the Next Generation Jammer. In 2014 Raytheon successfully demonstrated the active electronically scanned array (AESA) and gallium nitride $(\mathrm{GaN})$ technology prototype at the US "Patriot" anti-missile air defense radar system. This technology enables 360 degree sensing coverage in the future; it will expand the scope of defense, and to reduce the time of detection, identification and threat elimination. AESA technology based GaN will further improve reliability of the "Patriot" radar and decreased life-cycle costs.

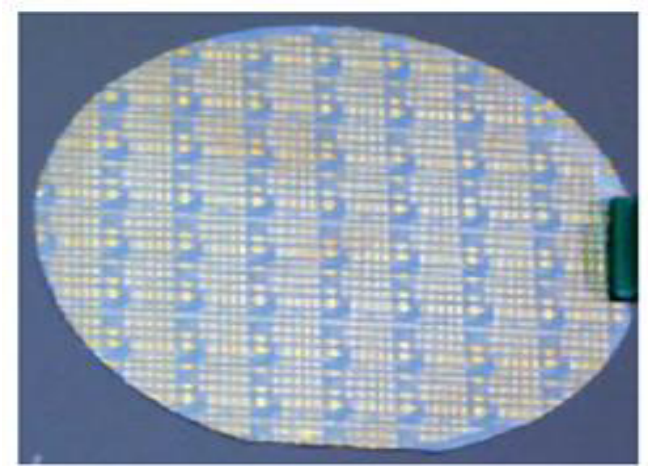

Figure 3. GaN MMIC developed by Raytheon Company for completing the second phase of WBGS

TriQuint Semiconductor, Inc. is a leader of GaN HEMT developed field, with the highest level of technology in the development of $\mathrm{GaN}$ alternative substrates, S-band and X-band GaN MMIC application has achieved excellent system performance. According to reports, the wide band-gap semiconductor SiC JFET has been able to replace conventional Si IGBT module in the US Navy's next-generation electric ships, such as DDG1000 systems. System simulation results show that, with the wide bandgap semiconductor $\mathrm{SiC}$, replacing $\mathrm{Si}$, many system parameters have been greatly improved. System power consumption can be reduced to $1 / 25$ of the original system; switching speeds improved 100 to 200 times than the past. In 2010, TriQuint Semiconductor, Inc. began to partici- 
pate in third phase project of the WBGS; in May 2014 it developed the device whose mean work time is more than 106 hours between failures at $200^{\circ}$ environment, made the successful completion of the third phase of the task. Base the research, many wide band-gap semiconductor devices were developed for radar.
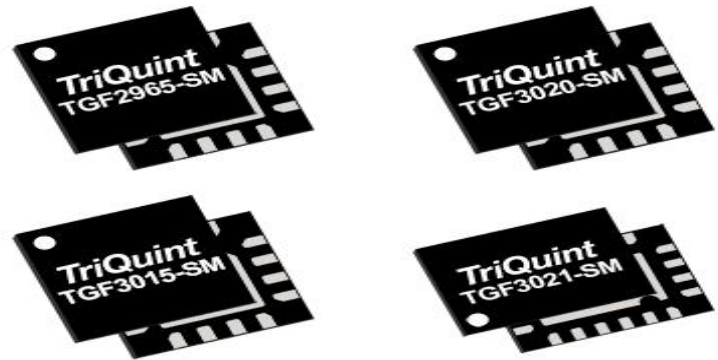

Figure 4. Wide band gap device for radar

$\mathrm{SiC}$ power modules developed by Cree. will be extensively used on the new aircraft of US Air Force F35LightingII. In power system applications, the efficiency of the power device is higher, the advantages of system will be more prominent, and reduce of carbon emissions is one of the obvious advantages. According to the difference of application areas, it will also bring many other benefits. If the wide band-gap power electronic devices are used in hybrid electric locomotive, DC battery power can be more efficiently converted to AC source; that can more effectively drive the engine and reduce energy consumption. Moreover, the heat dissipation of electronic components will be reduced, so that the cooling system will be smaller, lighter, less complex, and ultimately reduce the production cost, save package space, and achieve energy saving purpose. Improving the device efficiency may also bring a series of advantages for military aircraft and alternative energy systems, such as improving efficiency can reduce weight, reduce the requirements for the cooling system, which can extend and expand the flying distance and range of the aircraft.

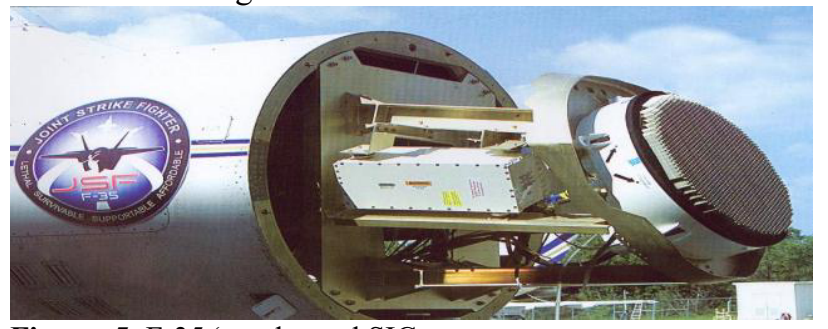

Figure 5. F-35's radar and SIC power components

As a leader in wide band gap technology, the United States has developed wide band-gap devices; in 2014, the M/A-COM Company has developed a new SiC-based GaN HEMT device, named MAGX-001214-650L00, this transistor operates in the L-band, $650 \mathrm{~W}$ peak power, can reliably operate at $50 \mathrm{~V}$ or even more extreme load adapt conditions. This device is shown in Fig. 2.

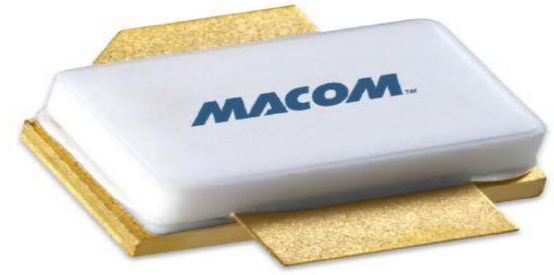

Figure 6. M / A-COM's power devices
In 2014, United States CREE Company also developed the CGHV1F006S and CGHV1F025S two power devices, two devices are the largest power continuous wave GaN HEMT in the field, use dual flat no-lead package. CGHV1F006S operating frequency covers DC-18GHz, output power is $6 \mathrm{~W}$; CGHV1F025S operating frequency covers DC- $18 \mathrm{GHz}$, output power is $25 \mathrm{~W}$.
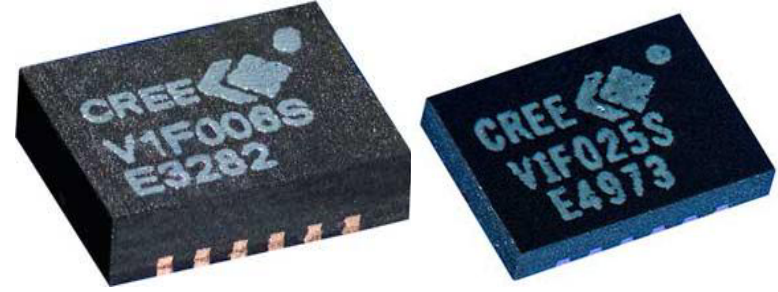

Figure 7. CREE Company developed two power devices

\section{3 SEMICONDUCTOR TECHNOLOGY}

The EU's military application intention of development wide band-gap technology is clear, in order to improve air defense surveillance radar and performance of the high RF wireless communication system, the EU focuses on efficient $1 \sim 40 \mathrm{GHz}$ frequency range, high power, high linearity and low cost single chip amplifier. The EU has developed the KORRIGAN plan, which is a five-year development plan in order to develop GaN HEMT technology in 2005. The EU hopes through carrying out the plan to make the European Union to form a complete industrial chain of wide band-gap semiconductor technology, which can contribute positively to the development and application of wide band gap semiconductor technology.

Seven countries of France, Italy, Netherlands, Germany, Spain, Sweden and the United Kingdom participate in KORRIGAN plan. Units participated the plan covers the technical field of materials research, circuit design, modeling studies, process control, reliability evaluation, heat treatment and packaging. In order to successfully achieve program objectives and develop advanced GaN technology in 2009 in accordance with established goals, KORRIGAN plan designed demonstration system specifically to verify the validity of GaN technology in a variety of applications, including S-band high-power amplifiers, $\mathrm{X}$-band broadband high-power amplifiers, low noise amplifiers and switches. In support of the EU government, the plan goes smoothly. Thus, in recent years the EU has made fruitful results in GaN HEMT devices and microstrip MMIC amplifiers research, performance of devices and circuits continues to improve, many technology level is highest in the world.

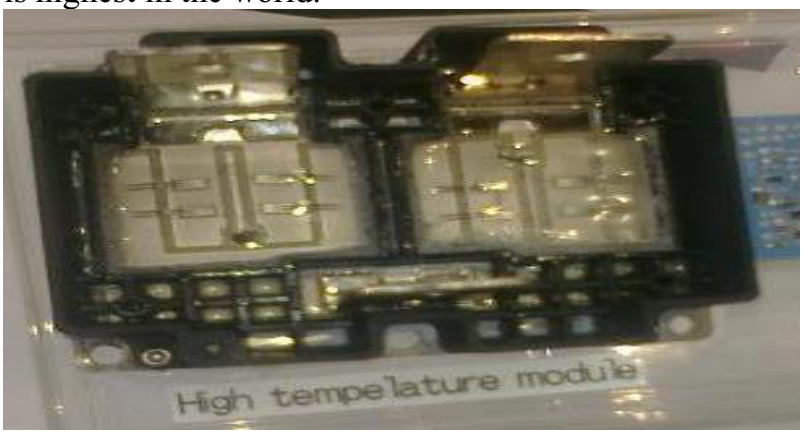

Figure 8. Germany developed GaN HEMT devices 
In 2010, under the leadership of the European Defence Agency, Germany, France, Italy, Sweden and the United Kingdom jointly launched "manufacture SiC substrate based $\mathrm{GaN}$ devices and $\mathrm{GaN}$ epitaxial layer wafer supply chain" (MANGA) project, the project aimed to optimize GaN-based power electronics R \& D and manufacturing, the project major research the GaN-based transistor layer epitaxial grown on newly developed $\mathrm{SiC}$ substrate, by using the existing OEM technology, these transistor layer are used in the manufacture of entirely European production HEMT. In the case of not depend on international suppliers, the EU five countries jointly developed a highquality GaN-based electronic devices, in 2014, the European Defence Agency declared that it has successfully established the supply chain for Europe GaN-based power electronics.

Saab Company has begun to develop GaN technology since 2005. The company will use GaN devices in its new Gripen active electronically scanned array (AESA) radar system. In May 2014, Saab Company announced the application scope of its Gripen active electronically scanned array radar system, including X-band and S-band three types land-based systems and two types sea-based systems. According the announce of Saab company, Gripen active electronically scanned array radar system 4A medium-range version and $8 \mathrm{~A}$ remote version are first batch of GaN complete two-dimensional system applied to a three-dimensional AESA multi-function radar, capable of simultaneous air defense, air surveillance and Weapon Locating.

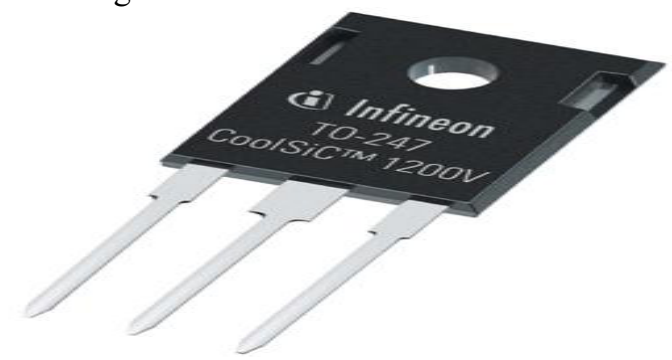

Figure 9. The SIC device of Infineon Company

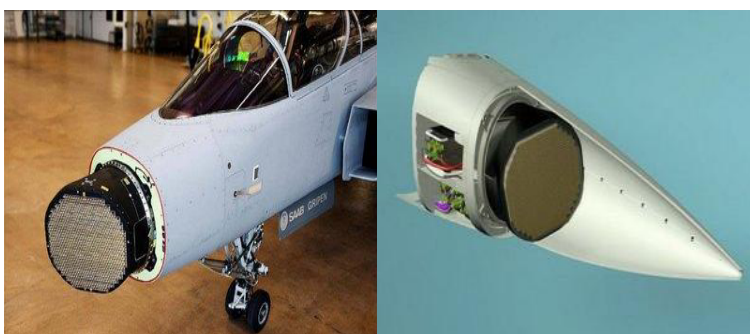

Figure 10. Gripen's active electronically scanned array radar system

\section{FACING CHALLENGE OF WIDE BAND- GAP TECHNOLOGY}

\subsection{Material immature or defective}

SiC single crystal material defect exists, then reduce and eliminate the defect density, and the increase of singlewafer size have become focuses of research. In recent years, great progress has been made in the respect of re- duction and eradication of fatal flaw microtubule density, which result the $\mathrm{SiC}$ power semiconductor performance and reliability decrease; Cree company began to supply 4 inches $\mathrm{SiC}$ single wafer of Zero Micropipes density in 2007. GaN material is immature, material defects results the critical breakdown field decline, Buffer substrate leakage of electricity is one of the main reasons of $\mathrm{GaN}$ power devices cannot reach the material theoretical limit.

\subsection{Reliability issues}

Two major technical difficulties of $\mathrm{SiC}$ devices have not been completely broken through: low inversion layer channel mobility and gate oxide reliability under hightemperature and high electric field. Through a special gate oxidation process, $\mathrm{SiC} / \mathrm{SiO} 2$ interface defects can be eliminated to improve the inversion layer channel mobility. For SiC BJT power devices, now an urgent need to address is the current gain degradation. The reasons of instability caused by the current gain are still unclear; one reason possibly is due to the stacking fault caused the epitaxial base region. Reliability issues exist in $\mathrm{GaN}$ device; trap, material defects, surface treatment and passivation layer protection, Buffer substrate leakage, degradation of Schottky gate metal under high voltage and high current and high field, insulated gate dielectric and surface charge and other issues impact the GaN device reliability.

\subsection{Packaging issues}

There are packaging problems in high-temperature, highpower $\mathrm{SiC}, \mathrm{GaN}$ device. When $\mathrm{SiC}, \mathrm{GaN}$ materials and processes problems are basically solved, Reliability issues of the device package will rise as the main factor impacting the high temperature high-power $\mathrm{SiC}, \mathrm{GaN}$ device performance. Especially GaN, with equal level of power density of $\mathrm{SiC}$, but the thermal conductivity is lower than $\mathrm{SiC}$; thus it exacerbates the problem of thermal spreading of $\mathrm{GaN}$ device, and puts forward a more serious challenge for high power $\mathrm{GaN}$ device package.

\section{CONCLUSION}

As the core technology of electronic, intelligence, integration and miniaturization of a new generation weapon, the third generation of semiconductor, that is wide band-gap semiconductor technology gets special attention of US, Japan, the European Union and other developed countries, for its set of advantages of small size, light weight, good stability, high reliability, low power consumption and so on. A number of wide band-gap semiconductor technology development programs implemented in these countries will make the wide band-gap semiconductor technology research continue to rise to new levels, so that a variety of wide band-gap semiconductor devices become satellite communications, high-speed computers, precisionguided, early warning and detection, intelligence and reconnaissance, electronic warfare, intelligent fire control systems and other military equipment's necessary and important components. 


\section{References}

1 CHOW $\mathrm{T}$ P. High-voltage $\mathrm{SiC}$ and $\mathrm{GaN}$ power devices[J]. Microelectronic Engineering, 2006, 83(1): 112-122.

2 TANAKA Y, OKAMOTO M, AKATSUKA A, et al. $700 \mathrm{~V} 1.0 \mathrm{~m} \Omega . \mathrm{cm} 2$ Buried Gate SiC-SIT (SiCBGSIT)[J]. IEEE Electron Device Letters, 2006, 27(11): 908-910.

3 MIKE C. Heterostructuring for high speed, power and light [J]. Semiconductor Today, 2009, 4(2): 7378.

4 YANAGIHARA M, UEMOTO A, UEDA T, et al. Recent advances in $\mathrm{GaN}$ transistors for future emerging applications[J]. Phys Status Solidi A, 2009, 206(6):1221-1227.

5 WONG King-yuen, CHEN WAN-JUN, CHEN KEVIN J. Integrated voltage reference and comparator circuits for $\mathrm{GaN}$ smart power chip technology $[\mathrm{C}] / /$ Proceedings of the 21th International Symposium on Power Semiconductor Devices \& IC's. Barcelona, Catalonia: IEEE, 2009: 57-60.

6 CHEN Wan-jun, WONG King-yuen, CHEN K J. Single-chip boost converter using monolithically integrated $\mathrm{AlGaN} / \mathrm{GaN}$ lateral field-effect rectifier and normally-off HEMT[J]. IEEE Electron Device Letters, 2009, 30(5):430-432

7 CHEN Wan-jun, WONG King-yuen, HUANG Wei, et al. High-performance AlGaN/GaN lateral fieldeffect rectifiers compatible with high electron mobility transistors [J]. Appl Phys Lett, 2008, 92(25): 253501.

8 ZHANG Bo, DENG Xiao-chuan, ZHANG You-run, et al. Recent development and future perspective of silicon carbide power devices-opportunity and challenge $[\mathrm{J}]$. Journal of China Academy of Electronics and Information Technology, 2009, 4(2): 111-118.

9 UEMOTO Y, HIKITA M, UENO $\mathrm{H}$, et al. Gate injection transistor(GIT) - A normally-off $\mathrm{AlGaN} / \mathrm{GaN}$ power transistor using conductivity
modulation[J]. IEEE Trans.Electron Devices, 2007, 54(12): 3393-3399.

10 HUANG W, LI Z; CHOW T P, et al. Enhancementmode GaN Hybrid MOS-HEMTs with Ron,sp of 20 $\mathrm{m} \Omega . \mathrm{cm} 2[\mathrm{C}]$. Proceedings of the 20 International Symposium on Power Semiconductor Devices \& IC's. Orlando, FL: IEEE,2008: 295-298.

11 SCHMELZER D,LONG S I.A GaN HEMT class F amplifier at $2 \mathrm{GHz}$ with $>80 \%$ PAE [J]. IEEE Journal of Solid-State Circuits,2007,42(10):2130-2136

12 RAMADAN A,REVEYRAND T,MARTIN A,et al.Experimental study on effect of second harmonic injection at input of classes $\mathrm{F}$ and $\mathrm{F}-1 \mathrm{GaN}$ power amplifiers [J].Electronics Letters,2010,46(8):570-572.

13 Mark Rosker, Christopher Bozada, Harry Dietrich, et al. The DARPA Wide Band Gap Semiconductors for RF Applications (WBGS-RF) Program: Phase II Results [C]. CS MANTECH Conference, 2009.

14 14Mark J. Rosker, John D. Albrecht, E. Cohen, et al. DARPA's GaN technology thrust [C]. Microwave Symposium Digest (MTT), 2010 IEEE MTT-S International , 2010:1214-1217.

15 Joe X. Qiu, A. M. Darwish, E. A. Viveiros, et al.Linearity characteristics study of millimeter-wave $\mathrm{GaN}$ power amplifier [C]. Microwave Symposium Digest (MTT), 2010 IEEE MTT-S International, $2011,1-4$,

16 Raymond S. Pengelly and Carl W. Janke. 1 to $2 \mathrm{GHz}$, 50 Watt Push-Pull Power Amplifier Using SiC MESFETs[J]. 2006 High Frequency Electronics. June 2006

17 Klimm D.Electronic materials with a wide band gap: recent developments[J]. IUCrJ. 2014, 9: 281-290.

18 Fabrizio Roccaforte, Patrick Fiorenza, Giuseppe Greco, et al. Challenges for energy efficient wide band gap semiconductor power devices[J], physica status solidi, 2014, 211(9): 2063-2071,

19 A. Hefner.Status of High-Voltage, High-Frequency Silicon-Carbide Power Devices[J]. GOMACTech 2006:232-235.

20 Rosker, M.J.Technologies for Next Generation T/R Modules [J].IEEE Radar Conference,2007:944 - 947 